\title{
Interaction of polycarboxylate-based superplasticiser with clay in Portland cement systems
}

\section{Guibo Gao}

Institute of Building Materials, China Academy of Building Research, Beijing, China; Key Laboratory of Pulp and Paper Science and Technology, Ministry of Education (Shandong Province), Qilu University of Technology, Jinan, Shandong, China

\section{Jun Ren}

Institute of Building Materials, China Academy of Building Research, Beijing, China; State Key Laboratory of Silicate Materials for Architectures (Wuhan University of Technology), Wuhan, Hubei, China (corresponding author: renjun@cabrtech.com)

\section{Yu Liu}

Key Laboratory of Pulp and Paper Science and Technology, Ministry of Education (Shandong Province), Qilu University of Technology, Jinan, Shandong, China

Jingyu Guo

Institute of Building Materials, China Academy of Building Research, Beijing, China

\section{Jun Li}

Institute of Building Materials, China Academy of Building Research, Beijing, China

It is widely accepted that polycarboxylate-based superplasticisers (PCEs) are sensitive to the presence of clay in cement and concrete. The impact of clay content on the adsorption behaviour of PCE on cement grain surfaces was investigated, with the adsorption of PCE on clay and cement grains determined by means of total organic carbon analysis. In addition, the effect of the mixing sequence of clay and PCE in cement paste was also investigated to explore the mechanism of the low clay-tolerance of PCE. The results indicated that the addition of clay reduced the adsorption of PCE on cement grain surfaces. Accordingly, the workability of the cement paste mixed with PCE was also changed with the addition of clay. In the presence of clay, although the total consumption of PCE was increased, the fluidity of the paste decreased. The results from different mixing sequences revealed that, in the presence of clay, the adsorption amount of PCE on cement grain surfaces was highly reduced, leading to poor dispersion of the cement particles.

\section{Notation \\ $C_{0} \quad$ initial concentration of superplasticiser (SP) solution $(\mathrm{mg} / \mathrm{ml})$ \\ $C_{\mathrm{t}} \quad$ concentration of SP solution after reaching adsorption equilibrium $(\mathrm{mg} / \mathrm{ml})$ \\ $c \quad$ equilibrium concentration of SP solution $(\mathrm{g} / \mathrm{l})$ \\ $k \quad$ adsorption constant $(\mathrm{l} / \mathrm{g})$ \\ $m \quad$ mass of binder $(\mathrm{g})$ \\ $R \quad$ gas constant $(8 \cdot 314 \mathrm{~J} / \mathrm{K} . \mathrm{mol})$ \\ $V \quad$ total volume of solution (ml) \\ $T \quad$ absolute temperature (K) \\ $\Gamma \quad$ adsorbed amount of SP per gram of binder $(\mathrm{mg} / \mathrm{g})$ \\ $\Gamma_{\mathrm{s}} \quad$ saturated amount of SP $(\mathrm{mg} / \mathrm{g})$ \\ $\Delta G_{\text {ads }} \quad$ adsorption free energy}

\section{Introduction}

Over the past 30 years, polycarboxylate-based superplasticisers (PCEs), known as the new generation of superplasticisers (SPs), have been shown to exhibit many advantages such as a high water-reducing ratio, low dosage requirement, good compatibility, low shrinkage, better slump retention and so on (Hirata, 1981). Due to their superb performance, PCEs have been successfully applied in many major construction projects. With increasing requirements on construction quality and demands for high-performance concretes, the need for PCEs will increase (Aitcin et al., 1994; Nematollahi and Sanjayan, 2014). However, many researchers have reported that PCE is quite sensitive to clay, which can be present as an impurity in aggregates (Fernandes et al., 2007; Ng and Plank, 2012; Sakai et al., 2006). The presence of clay has been reported to result in a rapid loss of workability or a higher water demand to achieve the same workability (Lei and Plank, 2012a; Tan et al., 2016a). Under such circumstances, no significant improvement in fluidity is observed for an increased dosage of PCE (Sakai et al., 2006; Sun, 2016; Tan et al., 2016b). Consequently, this sensitivity to clay negatively impacts the performance of PCE and, in turn, the workability and pumpability of concrete.

The mechanism of the compatibility between PCE and clay has been researched. Jeknavorian et al. (2003) reported that expanded clay adsorbed PCE and reduced the effectiveness of PCE. Sánchez-Martín et al. (2008) reported that more PCE was adsorbed on clay surfaces than on cement surfaces, due to the larger specific surface of the clay grains providing more adsorption sites. $\mathrm{Ng}$ and Plank (2012) revealed that PCE was adsorbed both chemically and physically on clay surfaces, with the adsorption amounts on clay approximately 100 times greater than on cement. Ferrari et al. (2010) suggested that the water-reducing effect of PCE is highly dependent on the surface charge of particles and the interactions between particles. Rao et al. (2009) found that PCE was adsorbed on particles through electrostatic attraction, ion exchange and hydrogen bonding and that the adsorption of PCE was affected by the type and chemical structure of the surfactant. Wang et al. (2010) reported that the amount of PCE adsorbed 
on kaolin surfaces was five times more than that on cement surfaces and the adsorption amount on bentonite was approximately 50 times more than that on cement. Lei and Plank (2014) reported that PCE is negatively affected by clays in the order montmorillonite $>$ kaolinite $>$ muscovite. However, these experiments were conducted using a single clay type whereas clay at construction sites is a mixture of all three clay types. Therefore, further study needs to be carried out for correct guidance on the usage of PCEs for on-site applications.

The main cause of the compatibility issue of PCE in the presence of clay can be summarised as competitive adsorption between cement and clay. Clays are hydrated aluminosilicates with crystal sizes typically less than $2 \mu \mathrm{m}$. Their crystal structures consist of alternating layers composed of sheets of silicon-centred tetrahedra and aluminium-centred octahedra. When dispersed in fluids containing ions, such as cement pore solution, cations can also adsorb on the negatively charged particle surfaces of clay grains ( $\mathrm{Ng}$ and Plank, 2012). Large amounts of PCE are thus adsorbed on clay grains, which consequently reduces the amount of PCE adsorbed on cement surfaces, leading to a poor PCE performance.

Based on this concept, adding extra PCE should significantly improve the fluidity of fresh concrete because more PCE is available for adsorption on cement. However, experiments have shown that the fluidity of fresh concrete is not obviously improved by increasing PCE dosage (Sakai et al., 2006), which suggests the need for further research. To understand the

Table 1. Physical properties of the PCE

\begin{tabular}{|lccc|}
$\begin{array}{l}\text { Solid } \\
\text { content: } \%\end{array}$ & $\begin{array}{c}\text { Density: } \\
\text { g/cm }\end{array}$ & $\begin{array}{c}\text { Surface } \\
\text { tension: MPa.s }\end{array}$ & $\mathbf{p H}$ \\
\hline 40.06 & 1.115 & 38 & 6.7 \\
\hline
\end{tabular}

Table 2. Chemical composition of raw materials

\begin{tabular}{lrr|} 
& Clay & Cement \\
\hline Silicon dioxide $\left(\mathrm{SiO}_{2}\right): \%$ & $64 \cdot 0$ & $23 \cdot 6$ \\
Aluminium oxide $\left(\mathrm{Al}_{2} \mathrm{O}_{3}\right): \%$ & $17 \cdot 0$ & $5 \cdot 1$ \\
Ferric oxide $\left(\mathrm{Fe}_{2} \mathrm{O}_{3}\right): \%$ & $5 \cdot 2$ & $2 \cdot 8$ \\
Calcium oxide $(\mathrm{CaO}): \%$ & $1 \cdot 5$ & $61 \cdot 7$ \\
Magnesium oxide $(\mathrm{MgO}): \%$ & $2 \cdot 5$ & $2 \cdot 2$ \\
Sodium oxide $\left(\mathrm{Na}_{2} \mathrm{O}\right): \%$ & $0 \cdot 4$ & $0 \cdot 1$ \\
Potassium oxide $\left(\mathrm{K}_{2} \mathrm{O}\right): \%$ & $1 \cdot 5$ & 0.8 \\
Sulfur trioxide $\left(\mathrm{SO}_{3}\right): \%$ & N/A & $2 \cdot 1$ \\
\hline
\end{tabular}

mechanism of the interaction between PCE and clay, the effect of clay on the adsorption behaviour of PCE on cement grain surfaces and the interaction between clay and cement was investigated in the work reported here.

\section{Experimental details}

\section{Materials}

The PCE was supplied by China Weihai Anlin Building Materials Co., Ltd. The number-average molecular weight and mass-average molecular weight of the PCE were 21800 and 42400 , respectively. The physical properties of the PCE are shown in Table 1.

The clay used in this work was clay extracted from river sand. The specific surface area of the clay was $10.86 \mathrm{~m}^{2} / \mathrm{g}$ and its chemical composition is shown in Table 2. The cement used was PO $42 \cdot 5$ cement from Yantai Mitsubishi Cement Co., Ltd. The chemical composition of the cement is shown in Table 2 and its properties are shown in Table 3.

\section{Sample preparation and mixing sequence}

For the workability tests, the water to cement ratio was fixed at $0 \cdot 30$ to enable direct comparisons and ensure reasonable paste workability. To explore the effect of clay on the workability of cement paste, three clay contents $(2 \cdot 0 \%, 6 \cdot 0 \%$ and $10 \cdot 0 \%)$ were investigated. The PCE dosage was set at $0 \cdot 38 \%, 0 \cdot 42 \%, 0 \cdot 46 \%$ or $0.50 \%$ by weight of the solid.

In order to assess adsorption, the liquid to solid ratio was fixed at 0.30 and the dosage of PCE was controlled at $0 \%$, $0 \cdot 125 \%, 0 \cdot 250 \%, 0.375 \%, 0.500 \%$ and $0.625 \%$ by weight of the solid. Four levels of clay content $(2 \cdot 0 \%, 4 \cdot 0 \%, 6 \cdot 0 \%$ and $100 \cdot 0 \%$ (clay)) were investigated.

To investigate the effects of the mixing sequence of the clay on PCE performance, three mixing sequences were studied

- simultaneous mixing (SM) - adding clay and PCE together when mixing with cement; the total mixing time was 9 min

n prior mixing (PM) - mixing cement with water first, adding clay at $3 \mathrm{~min}$ and then adding PCE at $6 \mathrm{~min}$ (Figure 1(a))

- delayed mixing (DM) - mixing cement with water first, adding PCE at $3 \mathrm{~min}$ and then adding clay at $6 \mathrm{~min}$ (Figure 1(b)).

Table 3. Properties of cement

\begin{tabular}{|c|c|c|c|c|c|c|}
\hline \multirow{2}{*}{$\begin{array}{l}80 \mu \mathrm{m} \\
\text { residual: \% }\end{array}$} & \multirow{2}{*}{$\begin{array}{l}\text { Specific surface } \\
\text { area: } \mathrm{m}^{2} / \mathrm{kg}\end{array}$} & \multirow{2}{*}{$\begin{array}{l}\text { Water demand } \\
\text { ratio: } \%\end{array}$} & \multicolumn{2}{|c|}{ Setting time: $\min$} & \multicolumn{2}{|c|}{ Compressive strength: MPa } \\
\hline & & & Initial & Final & $3 d$ & $28 d$ \\
\hline $2 \cdot 0$ & 335 & $26 \cdot 0$ & 130 & 190 & 18.9 & $48 \cdot 6$ \\
\hline
\end{tabular}




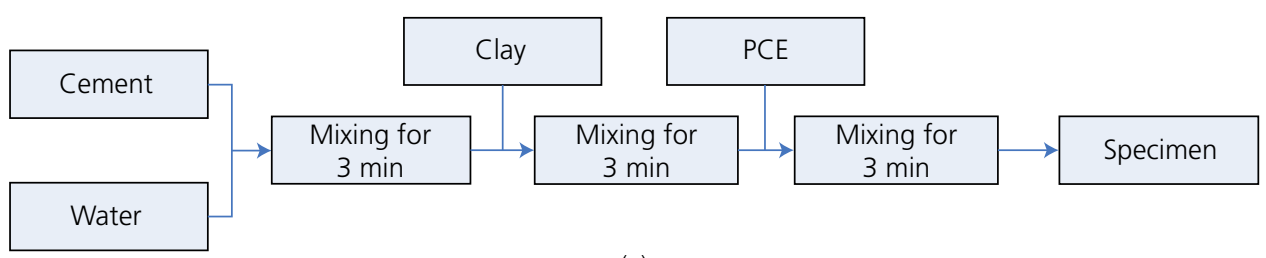

(a)

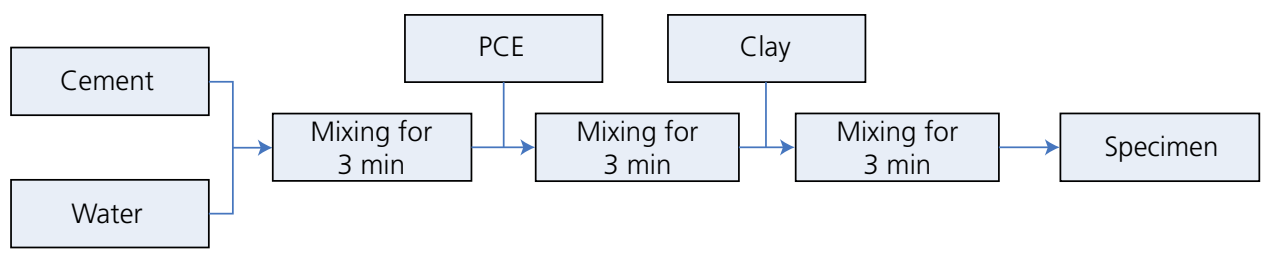

(b)

Figure 1. Mixing procedures: (a) PM; (b) DM

\section{Test procedures}

\section{Workability}

Slump flow tests carried out according to GB 8077 (SAC, 2012) were conducted to determine the workability of the pastes. A metal cone with a lower inner diameter of $60 \mathrm{~mm}$, an upper inner diameter of $36 \mathrm{~mm}$ and a height of $60 \mathrm{~mm}$ was used to determine the spread. Initial slump flow measurements were conducted $5 \mathrm{~min}$ after mixing (zero time). In order to test workability retention, the slump flow was again investigated after 1 to $8 \mathrm{~h}$ (depending on the setting of the cement).

\section{Adsorption}

Adsorption on cement was measured using the depletion method. In a typical experiment, $500 \mathrm{~g}$ of cement, $150 \mathrm{~g}$ of deionised water and the amount of PCE to be tested were placed in a $500 \mathrm{ml}$ container and stirred for $9 \mathrm{~min}$. The adsorption of PCE on clay and cement was determined using a total organic carbon (TOC) analyser (Shimadzu). The analysis was conducted on the aqueous phase, which was separated by two-stage centrifugation. The solution was firstly separated by centrifuging at $3500 \mathrm{rpm}$ for $15 \mathrm{~min}$ and then the supernatant from the first step was further centrifuged at $12000 \mathrm{rpm}$ for a further $15 \mathrm{~min}$. The supernatant was immediately decanted and diluted with deionised water for TOC analysis. The adsorption amount of PCE was calculated from the difference in PCE concentration before and after contact with the binder. The calculation is expressed as

1. $\Gamma=\frac{\left(C_{0}-C_{\mathrm{t}}\right) V}{m}$

where $\Gamma$ is the adsorbed amount of SP per gram of binder $(\mathrm{mg} / \mathrm{g}), C_{0}$ is the initial concentration of SP solution $(\mathrm{mg} / \mathrm{ml})$, $C_{\mathrm{t}}$ is the concentration of SP solution after reaching adsorption equilibrium $(\mathrm{mg} / \mathrm{ml}), V$ is the total volume of solution (ml) and $m$ is the mass of binder (g).

\section{Results}

\section{Effect of clay on the workability of cement paste}

Effect of clay content on cement paste workability

The effects of different clay contents on the initial slump flow of PCE-dosed cement pastes are shown in Figure 2. It is obvious that the addition of clay significantly decreased the slump flow of the pastes and the slump flow decreased with increasing clay content. For example, at $0 \cdot 50 \%$ PCE dosage, the slump flow of the cement paste decreased from $280 \mathrm{~mm}$ to $210 \mathrm{~mm}$ when mixed with $6 \%$ clay and further decreased to less than $150 \mathrm{~mm}$ after increasing the clay content to $10 \%$.

Noticeably, the clay content also exhibited a great influence on the performance of the PCE. It is obvious from Figure 2 that, at $2 \%$ clay content, increasing the PCE dosage from $0 \cdot 38 \%$ to

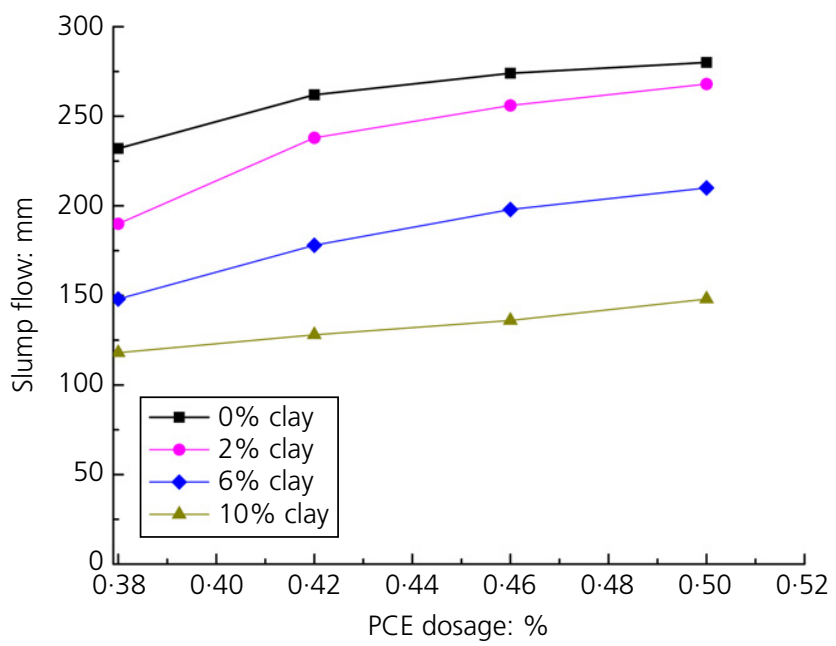

Figure 2. Effect of clay content on the workability of cement pastes mixed with PCE 
$0 \cdot 50 \%$ increased the slump flow by $34 \cdot 8 \%$. However, such a large improvement in slump flow with increasing PCE dosage was not seen for a clay content of $10 \%$, with this cement paste showing only a $25 \cdot 4 \%$ increase in slump flow when adding an extra $0 \cdot 12 \%$ PCE (from $0 \cdot 38 \%$ to $0 \cdot 50 \%$ ).

Other researchers have proposed that the competition between clay grains and cement grains for the adsorption of PCE is the main reason for the incompatibility of PCE with clay: adding extra PCE could eliminate the side effects of the clay by offering more PCE for adsorption onto cement surfaces, benefiting the dispersion of cement particles (Sakai et al., 2006; $\mathrm{Ng}$ and Plank, 2012). However, based on the results obtained here, for a higher content of clay, adding more PCE did not significantly enhance the workability of the cement paste, which indicates that the competition between clay and cement might not be the main reason for the dysfunction of PCE on the dispersion of cement particles in the presence of clay.

\section{Effect of mixing sequence on cement paste workability}

The effect of the mixing sequence of clay and PCE on the initial slump flow of the cement pastes is shown in Figure 3. The figure clearly shows that the mixing sequence had an impact on cement paste workability. When mixing clay to the mix before adding the PCE (PM), the initial slump flow of the cement paste obviously decreased with increasing clay content. With DM (adding PCE before clay), an increase in clay content had much less influence on the initial slump flow. However, a decrease in the adsorption amount of PCE on cement surfaces was observed for all mixing sequences, suggesting that clay reduces the adsorption of PCE.

To obtain deeper understanding of the effects of the mixing sequence, workability retention was investigated for a paste

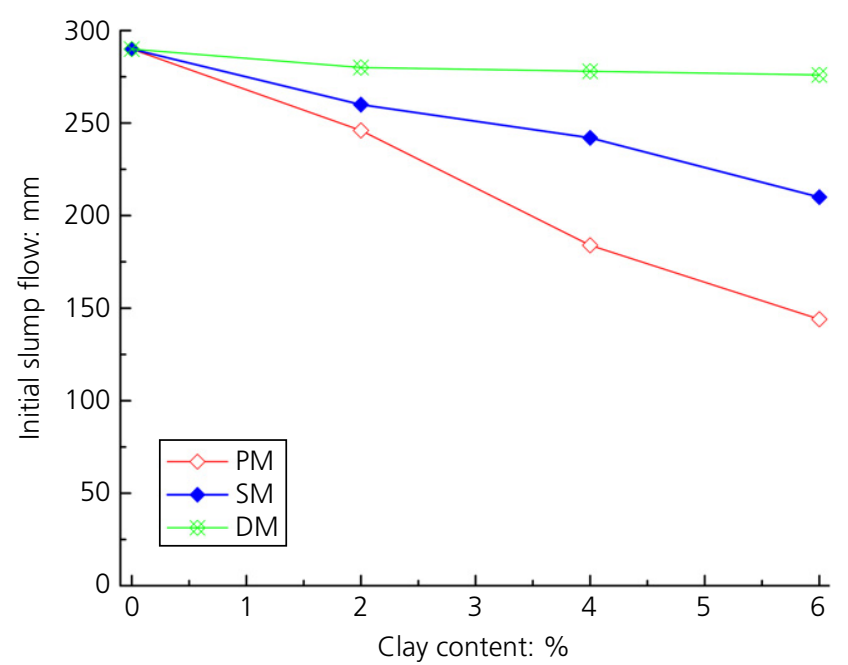

Figure 3. Effect of mixing sequence on the workability of cement pastes mixed with $0.5 \%$ PCE with no clay and cement paste with $6 \%$ clay prepared with the three different mixing sequences. The results in Figure 4 clearly show that the addition of clay led to poor workability retention. Regardless of the mixing sequence, the pastes with clay lost fluidity in less than $5 \mathrm{~h}$ while the paste without clay still showed flow after $8 \mathrm{~h}$. Figure 4 also shows that the mixing sequence had an impact on the workability retention of the PCE-dosed cement pastes: the poorest workability retention was observed with PM, but workability retention was improved by applying DM.

The major difference between PM and DM is the sequence of the contact of the clay and PCE with the cement. Once the clay grains contacted with the cement, the workability and workability retention of the cement paste was poor, which indicates that clay negatively affects the performance of PCE. However, the change in workability retention for the different mixing sequences suggests that the adsorption behaviour of the PCE was altered, indicating the need for adsorption tests.

\section{Effect of clay on the adsorption behaviour of PCE in cement paste}

The effects of clay on the adsorption behaviour of PCE are shown in Figure 5. It is clear that, compared with the adsorption on pure cement, the total adsorption of PCE increased in the presence of clay. For example, for a PCE dosage of $0.50 \%$, the adsorbed amount of PCE increased from $1.684 \mathrm{mg} / \mathrm{g}$ in the absence of clay to $3.219 \mathrm{mg} / \mathrm{g}$ in the presence of $6 \%$ clay - a $91 \%$ increase in PCE adsorption. This significant increase is due to the high adsorption of PCE on clay grains.

In a pure cement suspension, the adsorption of PCE on the surface of particles is strongly affected by two factors: $(a)$ the nature of the surface of the adsorbing substrate (its surface

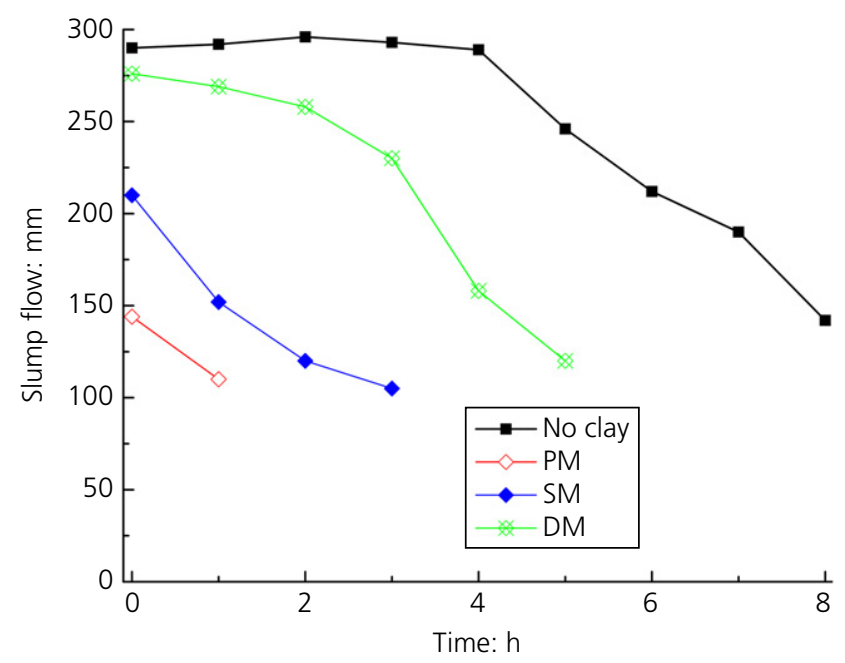

Figure 4. Effect of mixing sequence on the workability of cement paste with $0.50 \%$ PCE and $6 \%$ clay 


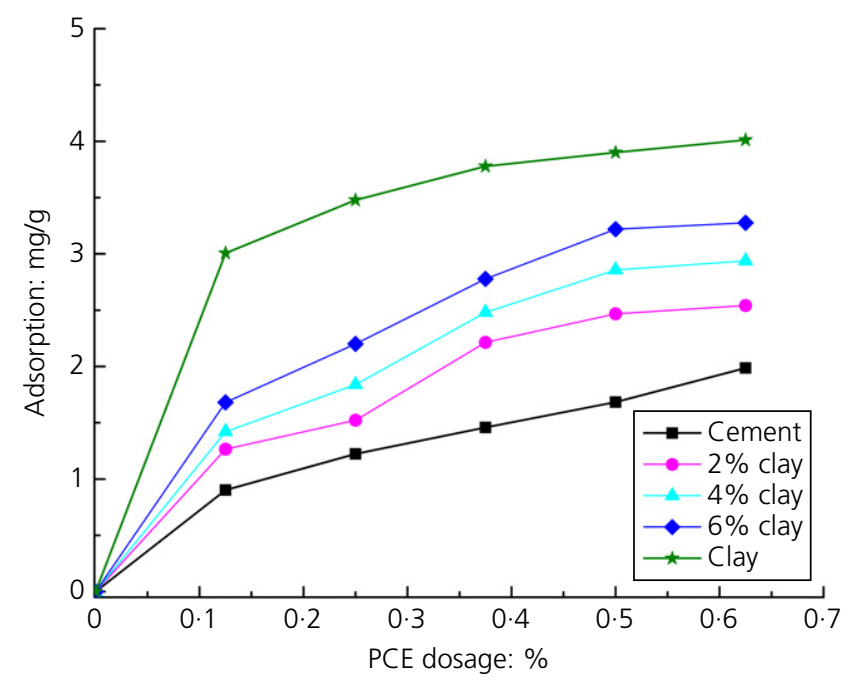

Figure 5. Effect of clay content on the adsorption behaviour of PCE

charge) and (b) the ionic conditions of the solution such as $\mathrm{pH}$ and ionic strength (Torn et al., 2003). Lei and Plank (2014) reported a negative surface charge on clay. As most of the PCE is an anionic polymer, the adsorption of PCE on clay should therefore be low. However, in this study, the adsorption amount of PCE increased in mixes with the addition of clay and the reason for this could be due to the high adsorption amount of PCE on the clay. As shown in Figure 5, for the same PCE dosage, the adsorption of PCE on pure clay grains was approximately $2 \cdot 5$ times that on cement particles; more PCE was thus adsorbed on the clay particle surfaces.

It is widely accepted that the adsorption behaviour of SPs follows Langmuir adsorption, which can be quantitatively expressed by (Peng et al., 2005)

2. $\Gamma=\Gamma_{\mathrm{s}} \frac{k c}{1+k c}$

3. $\frac{c}{\Gamma}=\frac{1}{\Gamma_{\mathrm{s}} k}+\frac{c}{\Gamma_{\mathrm{s}}}$

\section{4. $\Delta G_{\text {ads }}=-R T \ln k$}

where $\Gamma$ is the adsorbed amount of SP $(\mathrm{mg} / \mathrm{g}), \Gamma_{\mathrm{s}}$ is the saturated amount of $\mathrm{SP}(\mathrm{mg} / \mathrm{g}), c$ is the equilibrium concentration of SP solution $(\mathrm{g} / \mathrm{l}), k$ is the adsorption constant, $R$ is the gas constant $(8 \cdot 314 \mathrm{~J} / \mathrm{K} . \mathrm{mol})$ and $T$ is absolute temperature.

It is obvious from Figure 5 that the adsorption of PCE follows the trend of Langmuir adsorption. Based on Langmuir isothermal adsorption, quantitative analysis of the adsorption behaviour of PCE was conducted and the parameters are summarised in Table 4 . The high value of $R^{2}(>0.94$ in all cases) from this analysis suggests that the Langmuir adsorption equation can be used to describe the adsorption behaviour of PCE. As the adsorption characteristic plateau $\left(\Gamma_{\mathrm{s}}\right.$ in Equation 2) depends on the attraction force between the PCE and the adsorbent, a higher value of $\Gamma_{\mathrm{s}}$ indicates that a stronger attractive force exists between the two. Therefore, results in Table 4 suggest that with the addition of clay, higher attractive forces were formed and therefore more SPs were adsorbed on the particle surfaces. In addition to $\Gamma_{\mathrm{s}}$, which can be used to indicate the affinity of a dispersant on cement surfaces, the Langmuir adsorption constant $k$ in Equation 2 can be used to calculate the adsorption free energy $\Delta G_{\text {ads }}$ in Equation 4. As shown in Table $4, \Delta G_{\text {ads }}$ for PCE on the surface of cement with/without clay was negative, which indicates that the adsorption of PCE on cement is favourable and the adsorption process is spontaneous.

Additionally, in the presence of both clay and cement, the total PCE adsorption amount was different from the theoretical calculations for a single-component paste. For example, at $0.5 \%$ PCE dosage, for each $100 \mathrm{~g}$ of cement paste (with $6 \%$ clay), it can be calculated from the data shown in Figure 5 that the adsorption of PCE on $94 \mathrm{~g}$ cement was $158 \cdot 3 \mathrm{mg}$ and that on $6 \mathrm{~g}$ of clay was $23.4 \mathrm{mg}$. Thus the total adsorption amount for $100 \mathrm{~g}$ paste was $181.7 \mathrm{mg}$. However, in the experiments, the adsorption amount of PCE for each $100 \mathrm{~g}$ cement paste with $6 \%$ clay was determined to be $321 \mathrm{mg}$. These results indicate that there is an interaction between clay and cement that impacts the adsorption behaviour of PCE and causes the incompatibility issues between PCE and clay.

Table 4. Parameters in the Langmuir equation for the adsorption of PCE on cement and clay

\begin{tabular}{|c|c|c|c|c|c|c|}
\hline Mixes & $R^{2}$ & Intercept & Slope & $k: \mathbf{l} / \mathbf{g}$ & $\Gamma_{s}: \mathbf{m g} / \mathbf{g}$ & $\Delta G_{\text {ads }}: \mathrm{kJ} / \mathrm{mol}$ \\
\hline Cement & 0.9585 & $272 \cdot 1$ & 355.9 & $1 \cdot 308$ & $2 \cdot 810$ & -0.6542 \\
\hline Clay & 0.9997 & $33 \cdot 7$ & $228 \cdot 0$ & 6.756 & $4 \cdot 387$ & $-4 \cdot 6564$ \\
\hline Cement $+2 \%$ clay & 0.9432 & $191 \cdot 0$ & $266 \cdot 1$ & $1 \cdot 393$ & 3.758 & -0.8085 \\
\hline Cement $+4 \%$ clay & 0.9712 & $165 \cdot 0$ & $230 \cdot 8$ & $1 \cdot 399$ & $4 \cdot 333$ & -0.9180 \\
\hline Cement $+6 \%$ clay & 0.9851 & 128.9 & $219 \cdot 1$ & 1.700 & $4 \cdot 563$ & $-1 \cdot 2928$ \\
\hline
\end{tabular}

The adsorption constant $k$ was obtained from the slope/intercept from the straight line in the plot of $1 / \Gamma$ against $1 / c$, and the saturated amount of SP $\left(\Gamma_{\mathrm{S}}\right)$ was calculated from $1 /$ slope 
Interaction between clay and PCE adsorption on cement

PCE is a type of comb-shaped surfactant with an anionic backbone and non-polar chain. After it dissolves in water, the hydrolysed SP is first adsorbed on particle surfaces and then particles are dispersed through electrostatic repulsion and steric repulsion (Ran et al., 2010). In electrostatic repulsion, the negatively charged SP is adsorbed onto the positively charged surface of cement particles by electrostatic attraction. The residue's negative charge contributes to the repulsion and dispersion of the cement particles by like- poles action. In steric repulsion, in addition to the electrostatic repulsion generated from the negative charges on the backbone of the SP, the non-charged long side chain physically keeps cement particles apart (Yamada et al., 2001). The working mechanism of PCE in Portland cement usually involves three steps: (a) adsorption of the SP on the surface of cement particles, $(b)$ dissolution in the aqueous phase and (c) intercalation, co-precipitation or micellisation within the hydration product (e.g. calcium silicate hydrate gel), forming an organo-mineral phase. Among these, the adsorption of the SP on the surface of the cement particles is a crucial stage for the dispersion of cement particles through electrostatic repulsion and/or steric repulsion (Flatt and Houst, 2001). Therefore, the more SP adsorbed on the cement particles, the better the dispersion. It should be noted that dissolved PCE in the pore solution could be adsorbed on the surface of the newly formed hydration product, continually contributing to the dispersion of cement particles, which retains workability along with the hydration.

Clay grains are a type of negatively charged mineral. In order to maintain electrical neutrality, many cations from the bulk solution are adsorbed on the surface of clay particles (Lei and Plank, 2012b). Therefore, $\mathrm{Ca}^{2+}$ ions released during hydration are more easily adsorbed onto the surface of clay grains, which benefits the adsorption of PCE on clay surfaces through electrostatic attraction. Therefore, a higher PCE adsorption amount is found on the clay surfaces. In addition, intercalation of the long polyethylene oxide side chains of PCE into the interlayered space of clay is thought to be another reason for its huge adsorption amount (Tan et al., 2016b). All these effects will reduce the dispersion efficiency of PCE on cement particles.

As illustrated in Figures 6 and 7, the adsorption of the clay reduces the positively surface charge density of cement, which provides less sites for PCE to be adsorbed. However, when adding more calcium nitrate, the positively charged $\mathrm{Ca}^{2+}$ provides more activated sites, and consequently, the adsorption amount of PCE was increased. Therefore, it can be concluded that, in the presence of clay, the adsorption of the PCE on cement particles is reduced (as shown in Figure 7), and hence, the efficiency for water-reducing effect of PCE is consequently reduced.

\section{Conclusion}

The effects of clay content on the performance of PCE in cement paste and the effects of the mixing sequence were

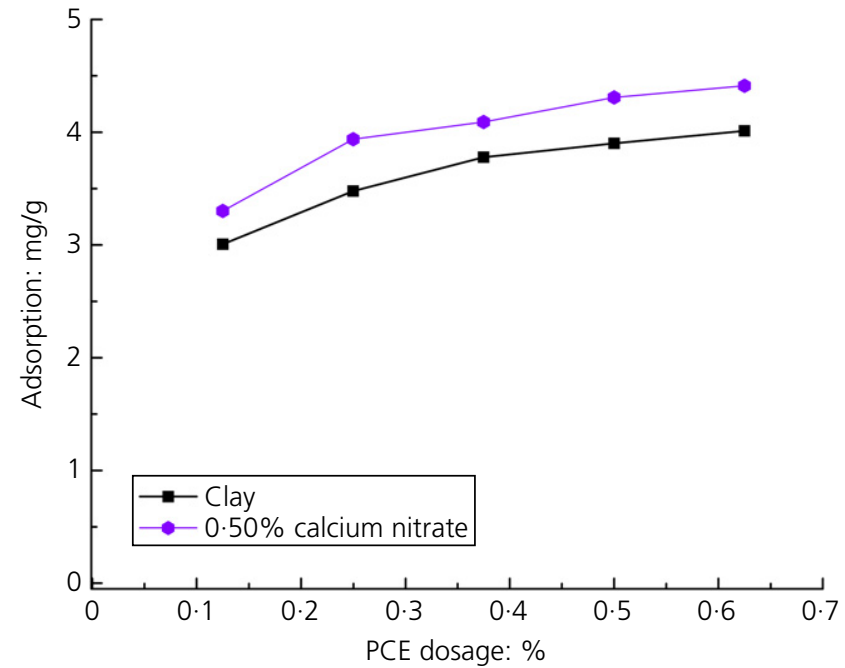

Figure 6. Effect of calcium nitrate on the adsorption behaviour of PCE

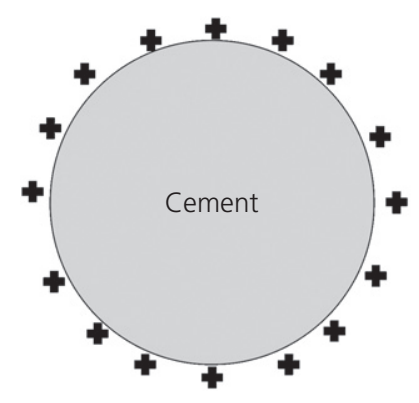

(a)

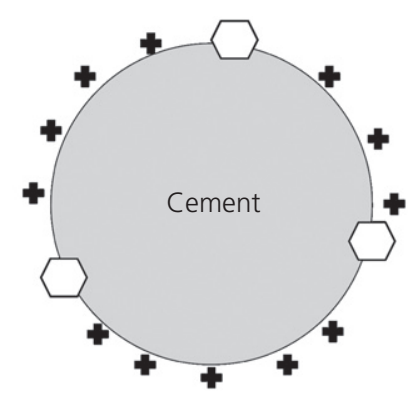

(b)
Figure 7. Schematic illustration of the effects of clay on the surface charge of cement particles in the absence of clay (a) and the presence of clay (b)

investigated. The following conclusions can be drawn from this work.

(a) The workability of cement paste dosed with PCE was significantly reduced with the addition of the clay. The workability of the cement paste with added clay was not obviously improved by increasing the PCE dosage.

(b) The mixing sequence of clay and PCE affected the workability and workability retention of the pastes. Compared with DM (adding PCE before clay), lower slump flow spreads were observed for PM (adding clay before PCE) and SM (adding clay and PCE together), with slump flow lower for PM than for SM. Of the three mixing sequences, DM produced the best workability retention.

(c) The adsorption of PCE was higher in paste mixed with both clay and cement than in paste comprising a single component. 
(d) The addition of clay reduced the efficiency of the PCE in terms of the dispersion of cement particles in two ways. Firstly, competition between the clay and the cement for the adsorption of cations in the bulk solution provided less adsorption sites on the cement surfaces, thus decreasing the adsorption amount of PCE on cement. Secondly, the consumption of PCE through adsorption and intercalation with the clay reduced the adsorption amount of PCE on cement.

\section{Acknowledgements}

The authors would like to acknowledge the Planned Project of Science and Technology of the Ministry of Housing and Urban-Rural Development of the People's Republic of China (2012-K4-7), the Opening Foundation of the Key Laboratory of Pulp and Paper Science and Technology, Ministry of Education (KF201413) and the Opening Foundation of the State Key Laboratory of Silicate Materials for Architectures (Wuhan University of Technology) (SYSJJ2017-16) for collective funding of this work.

\section{REFERENCES}

Aitcin PC, Jolicoeur C and Macgregor JG (1994) Superplasticizers: How they work and why they occasionally don't. Concrete International 16(5): 45-52.

Fernandes VA, Purnell P, Still GT and Thomas TH (2007) The effect of clay content in sands used for cementitious materials in developing countries. Cement and Concrete Research 37(5): 751-758.

Ferrari L, Kaufmann J, Winnefeld F and Plank J (2010) Interaction of cement model systems with superplasticizers investigated by atomic force microscopy, zeta potential, and adsorption measurements. Journal of Colloid and Interface Science 347(1): 15-24.

Flatt RJ and Houst YF (2001) A simplified view on chemical effects perturbing the action of superplasticizers. Cement and Concrete Research 31(8): 1169-1176.

Hirata T (1981) A Cement Dispersant. JP Patent 84, 2022, S59-018338, Apr.

Jeknavorian A, Jardine L, Ou C, Koyata H and Folliard K (2003) Interaction of superplasticizers with clay-bearing aggregates. Proceedings of the 7th Canmet/ACI International Conference Superplasticizers and Other Chemical Admixtures in Concrete. ACI, Farmington Hills, MI, USA, ACI SP 173-04, pp. 55-82.

Lei $L$ and Plank J (2012a) A concept for a polycarboxylate superplasticizer possessing enhanced clay tolerance. Cement and Concrete Research 42(10): 1299-1306.

Lei L and Plank J (2012b) Synthesis, working mechanism and effectiveness of a novel cycloaliphatic superplasticizer for concrete. Cement and Concrete Research 42(1): 118-123.
Lei L and Plank J (2014) A study on the impact of different clay minerals on the dispersing force of conventional and modified vinyl ether based polycarboxylate superplasticizers. Cement and Concrete Research 60(6): 1-10.

Nematollahi B and Sanjayan J (2014) Effect of Superplasticizers on Workability of Fly Ash Based Geopolymer, Springer, Singapore.

$\mathrm{Ng} \mathrm{S}$ and Plank J (2012) Interaction mechanisms between $\mathrm{Na}$ montmorillonite clay and MPEG-based polycarboxylate superplasticizers. Cement and Concrete Research 42(6): 847-854.

Peng J, Qu J, Zhang J, Chen M and Wan T (2005) Adsorption characteristics of water-reducing agents on gypsum surface and its effect on the rheology of gypsum plaster. Cement and Concrete Research 35(3): 527-531.

Ran Q, Somasundaran P, Miao C et al. (2010) Adsorption mechanism of comb polymer dispersants at the cement/water interface. Journal of Dispersion Science \& Technology 31(6): 790-798.

Rao P, Xu L, Zhang W and Xiao W (2009) Review of adsorption of surfactants on soil particles and its effect factors. Journal of Anhui Agricultural Sciences 37(14): 6542-6545.

SAC (Standardization Administration of China) (2012) GB 8077-2012: Methods for testing uniformity of concrete admixture. Standardization Administration of China, Beijing, China.

Sakai E, Atarashi D and Daimon M (2006) Interaction between superplasticizers and clay minerals. In Proceedings of the 6th International Symposium on Cement \& Concrete and Canmetl ACI International Symposium on Concrete Technology for Sustainable Development. The Chinese Ceramic Society, Beijing, China, vol. 2, pp. 1560-1566.

Sánchez-Martín MJ, Dorado MC, Hoyo CD and Rodr Guez-Cruz MS (2008) Influence of clay mineral structure and surfactant nature on the adsorption capacity of surfactants by clays. Journal of Hazardous Materials 150(1): 115-123.

Sun SM (2016) A review for interaction mechanism between polycarboxylate superplasticizers and clay. Guangzhou Architecture 44(1): 33-35 (in Chinese).

Tan H, Qi C, Ma B, Li X and Jian S (2016a) Effect of polycarboxylate superplasticiser adsorption on fluidity of cement-clay system. Materials Research Innovations 19(sup5): S5-423-S5-428.

Tan H, Gu B, Ma B et al. (2016b) Mechanism of intercalation of polycarboxylate superplasticizer into montmorillonite. Applied Clay Science 129: 40-46, https://doi.org/10.1016/j.clay. 2016.04.020.

Torn LH, Keizer AD, Koopal LK and Lyklema J (2003) Mixed adsorption of poly (vinylpyrrolidone) and sodium dodecylbenzenesulfonate on kaolinite. Journal of Colloid and Interface Science 260(1): 1-8.

Wang Z, Cheng X and Li D (2010) Effects of different clay to the application performance of high range water reducer. Ready-mixed Concrete 7(3): 24-26 (in Chinese).

Yamada K, Ogawa S and Hanehara S (2001) Controlling of the adsorption and dispersing force of polycarboxylate-type superplasticizer by sulfate ion concentration in aqueous phase. Cement and Concrete Research 31(3): 375-383.

\section{How can you contribute?}

To discuss this paper, please submit up to 500 words to the editor at journals@ice.org.uk. Your contribution will be forwarded to the author(s) for a reply and, if considered appropriate by the editorial board, it will be published as a discussion in a future issue of the journal. 